\title{
A protocol for the aerial survey of penguin colonies using UAVs
}

Norman Ratcliffe, Damien Guihen, Jeremy Robst, Sarah Crofts, Andrew Stanworth and Peter Enderlein

N. Ratcliffe, D. Guihen, J. Robst, P. Enderlein. British Antarctic Survey, High Cross, Madingley Road, Cambridge, CB25 OEF, UK.

Sarah Crofts, Andrew Stanworth. Falklands Conservation, Jubilee Villas, Ross Road, Stanley, Falkland Islands, FIQQ $1 Z Z$

Abstract: Penguins, and many other seabirds, often nest in the open in large colonies, and so are amenable to aerial survey. UAVs offer a flexible and inexpensive method of achieving this but, to date, few published examples are available. We present a protocol for acquiring aerial images of penguin colonies using UAVs and describe simple, open-source tools for processing these into counts. Our approach is demonstrated using a case study for a penguin colony in the Falkland Islands. We discuss the advantages and limitations of UAVs for penguin surveys and make recommendations for their wider application.

Keywords: aerial survey, colony, drone, penguin, seabird, UAV.

\section{Introduction}

Counts of penguins are important to monitor population sizes and trends for conservation purposes and to better understand their importance in food-webs (Lynch et al. 2012a). Penguin surveys have mostly been based on counts of nests from the ground during incubation; either by direct head-counts for smaller colonies or by sampling nesting density and extrapolating this to the colony area for large ones (Lynch et al. 2008). These methods may have substantial count or sampling errors, can be very time-consuming and create disturbance to penguins if observers enter 
the colony. More recently, aerial surveys of penguin colonies from manned aircraft and surveys from satellites have been attempted with some success (Fretwell et al. 2012; Lynch et al. 2012b; Trathan et al. 2012). However, conventional aerial surveys are expensive, potentially dangerous and may be obliged to fly at high altitudes to avoid disturbance to penguins (with associated loss of image quality), while the resolution of satellite surveys is currently limited to estimating the area of occupation rather than counting individual nests.

The use of unmanned aerial vehicles (UAVs) in ecology has proliferated over the past decade as they offer an inexpensive, portable and low-disturbance platform for aerial survey of animals (Watts et al. 2010; Anderson and Gaston 2013; Vermeulen et al. 2013), their habitats (Koh and Wich 2012; Chabot and Bird 2013) and threats to both (Merino et al. 2011; Mulero-Pázmány et al. 2014) . Many seabirds, including most penguin species, nest in the open in large colonies and so have enormous potential for survey by UAVs. Despite this, there are few examples of the application of UAVs to seabird surveys: an extensive web search found only three popular articles (Gardner et al. 2010; Marquis 2013; Hodgson and Clarke 2014) and three published papers (Sardà Palomera et al. 2012; Grenzdöffer 2013; Goebel et al. 2015). Further published examples explaining the protocols for UAV surveys of seabird colonies are needed to promote the wider application of this technique.

This paper describes a survey of a penguin colony using a UAV. We describe the equipment we used, outline a protocol for survey work and describe a simple approach to image analysis using open-source software. We describe the advantages and limitations of UAVs for penguin censuses and make recommendations for their application to survey work in the future.

\section{Methods}

\subsection{Study site and species}

The study site was Volunteer Point (Lat -51.48, Lon:-57.83): an isthmus in the NE corner of East Falkland that hosts several gentoo penguin sub-colonies. It is accessible from the main settlement, Stanley, by a four-hour drive in a $4 \times 4$ vehicle. Gentoo penguins Pygoscelis papua are widespread 
through the Subantarctic and on the Antarctic Islands and Peninsula, generally occurring in relatively small colonies which are of a manageable size for preliminary UAV trials. The fieldwork extended from 17 Nov to 1 Dec 2014, a period when gentoo penguins were mostly incubating eggs, with some just beginning to hatch their chicks.

\subsection{Platform}

As penguin colonies are often in rugged and rocky environments the availability of natural runways cannot be relied upon, so we selected multi-rotor UAVs as our aerial platform for their vertical take-off and landing ability. The pilot (NR) trained for a year using the AeroSim RC flight simulation package and a DJI Naza F550 hexacopter before moving on to a further year of training using the UAV that was used in the survey. This comprised a Cinestar 6 carbon fibre airframe equipped with Microcopter electronics and six Quadrocopter motors, a maximum payload of $2 \mathrm{~kg}$ (Fig. 1). The UAV was controlled with a Spektrum DX8 handset. The Microcopter system includes a number of features that facilitate easier flying, including altitude hold, position hold and autopilot flight along a series of pre-defined waypoints or to the point of takeoff. Communication between the UAV was maintained using a ground station comprising an XBee radio link mounted on a tripod and connected to a laptop (Fig. 2). This allowed flight-data from the UAV to be viewed in real time on the laptop screen and programming of the UAV's navi-control with waypoints for flying in auto-pilot. We also had the option of live-feed video from a miniature FPV camera fitted to the UAV which aided visual alignment with targets during flight.

A Canon EOS M mirrorless SLR with a $22 \mathrm{~mm}$ pancake lens was used for photography. This has an APS-C sensor which produces high quality images while being small and light enough to be carried easily by the UAV. This was mounted directly onto the airframe with an $L$ shaped aluminium bracket so it faced straight downwards. To minimise blurring due to movement of the airframe, we set the lens aperture to $\mathrm{f} / 2.2$ which ensured a fast shutter speed. The open-source firmware Magic Lantern (2013) was downloaded onto the camera's SD card. This increases the functionality of the EOS M, 
including the facility of time-lapse photography that was required for the camera to shoot a series of images automatically while the UAV was in flight. Images were shot at the highest possible quality in jpeg format, with each frame having a size of c. $12 \mathrm{Mb}$.

\subsection{Survey Protocol}

A flight altitude of $30 \mathrm{~m}$ gave excellent resolution images of penguins (Fig. 3). At this altitude birds incubating eggs or attending their chicks did not leave their nest, although gentoo penguins and nearby king penguins Aptenodytes patagonicus that were not engaged in nesting activity would walk away from the UAV when it was overhead. A speed of $5 \mathrm{~m} / \mathrm{s}$ avoided blurring of images while ensuring a reasonable pace of progression along the flight-path. From this altitude, the field of view was $30 \mathrm{~m}$ wide and $20 \mathrm{~m}$ high, as calculated using an online lens calculator (Points in Focus, 2010). We allowed a $5 \mathrm{~m}$ overlap in images along each axis to avoid the risk of gaps between photographs due to deviations from the flight path. Transects were therefore spaced $25 \mathrm{~m}$ apart and the shutter was set to release every $3 \mathrm{~s}$.

In preparation for the survey, the UAV was switched on and carried around the edge of the colony: the GPS track relayed back to the ground station produced a map of the colony perimeter on the screen of the laptop. Transects of the required length, orientation and spacing were then constructed automatically in a rectangle across this area using the Microcopter software, and their endpoints were dragged to the colony perimeter with the laptop's mouse to avoid overflying areas without penguins. The waypoints were then uploaded to the navi-control of the UAV and, after launch, it was missioned to follow transects on autopilot. The heading of the UAV was kept constant throughout the flight.

As a contingency against failure of the autopilot function, we could resort to manual flying according to the Perryman method (Gardner et al. 2010). This required the pilot and an assistant to stand on opposite sides of the colony, starting at one end of it. The UAV was then launched, flown to 
$30 \mathrm{~m}$ altitude and put on altitude hold. The pilot flew the UAV to the assistant at approximately $5 \mathrm{~m} / \mathrm{s}$, and the assistant indicated when it was overhead. Both then walked $25 \mathrm{~m}$ along the colony perimeter. The UAV was then flown over to the assistant's new position and then in a straight line back to the pilot. This process is repeated until the entire colony area has been surveyed.

\subsection{Image processing}

Images were stitched into a single mosaic using Image Composite Editor (Microsoft Research 2014). This was compressed to $20 \%$ of its original size using GIMP (2014) as it was too large (312Mb) to be loaded into the counting software. Penguins in the image were counted using iTag (Viquerat and van Neer 2014). All three of the image-processing packages are available open-source. iTag allows the user to place different colour dots on targets in the image that have different attributes. In our case, we marked birds incubating eggs or brooding chicks in a different colour to those standing around or in the colony. The software returns a spreadsheet of the total count of dots of each colour in the image upon request.

During the fieldwork period, a direct count of active nests in all Volunteer Point gentoo penguin sub-colonies was conducted by two experienced fieldworkers from the ground, allowing comparisons to be made between the methods. The observers made repeated counts of the colony until their totals were within $10 \%$ of each other.

\section{Results}

Due to persistent strong winds ( $>12 \mathrm{~m} / \mathrm{s}$ ) and occasional precipitation, conditions were only suitable for flying during four days over the three week field season, and never for the whole of even these days. The long travel time to Volunteer Point meant that taking advantage of the relatively short weather windows that were suitable for flying was difficult. However, conditions were suitable on the morning of 27 Nov while we were camped at Volunteer Point and so a survey was conducted then. 
Despite having performed perfectly during tests in Stanley, the UAV developed a magnet error during the actual survey. Recalibration of the compass failed to rectify this, so the error was most likely due to electrical interference from the power cables and distribution board (an intermittent fault often reported online in Microcopter forums that can be fixed by shielding or moving the navigation control board). The magnet error resulted in the UAV flying erratically when in position hold and autopilot modes. We therefore resorted to manual flying using the Perryman method. Once the UAV was set up, the survey of one of the largest sub-colonies at Volunteer Point only took five minutes.

We successfully acquired images that covered the entire colony, and these were stitched into a mosaic (Fig. 4). A count from the image produced a total of 1,021 active nests and 207 birds standing in the colony, compared to a ground count of $1,327 \pm 23$ active nests.

\section{Discussion}

Our survey and those by Goebel et al. (2015) show that it is possible to obtain high quality images of penguin colonies using UAVs for relatively low effort. The accuracy of these counts is greater than that from ground counts, and a permanent record of the colony is maintained that can be checked for accuracy in the future. In this study, the nest counts from the ground were higher than those from the air as also found by Goebel et al. (2015): it seems likely that this arose from double-counting or standing birds being mis-assigned as being on nests. The aerial photographs allowed careful inspection and marking of every single bird in the colony, which meant such errors were eliminated. Aerial surveys also have the potential to yield valuable spatial information about the colony, including its location, shape, area, nest spacing and habitat type. For example, Grenzdörffer (2013) produced a digital elevation model of a common gull Larus canus colony, allowing nesting density to be related to topography and vegetation structure.

The drawback of UAVs is that weather has to be relatively calm and dry for flying, and such conditions are rare in the locations where penguins breed. Goebel et al. (2015) state a maximum 
wind speed of $<10 \mathrm{~m} / \mathrm{s}$ for safe UAV operations, which accords with our own observations. Due to strong winds we only succeeded in surveying one colony in two weeks, whereas 20 colonies in NE Falkland were counted in three days from the ground. Gardner et al. (2010) found only half of the days were suitable for UAV surveys of penguin colonies at Cape Sherriff, South Shetland Islands, and then only for part of those days. Precipitation also prevents UAV operations owing to risks to the exposed electronics, but trials at Halley station on the Antarctic continent (Lat -75.58, Lon:-26.65) show freezing temperatures have little effect apart from a modest reduction in battery life (A. Cziferszky, pers. comm.).

UAVs can also be prone to malfunctions or crashes which may curtail survey work. The magnet errors in our survey resulted in significant lost time and prevented the survey of more colonies, while our attempt to survey Adélie penguins Pygoscelis adeliae colonies on Signy Island, South Orkneys, in 2013 was bought to an abrupt end when the UAV crashed in the sea. Issues relating to safety and legislation may also limit the use of UAVs for seabird surveys in some locations (e.g. Fox News 2012).

UAVs are bulky, fragile and need to be kept dry during transportation. This was not a problem for our study site as it was accessible by vehicle, but where sites are accessed by foot or in open boats the safe transportation of a UAV becomes problematic. UAVs are therefore most effective where fieldworkers reside next to penguin colonies so that they only need to be transported for short distances and can be deployed or retrieved rapidly in response to changing weather conditions. Obtaining surveys during short, opportunistic visits by foot or from ships would require a small, portable UAV and considerable luck with the weather. However, UAVs may be useful during such visits by allowing ground-counts to be supplemented with aerial surveys, when conditions allow.

We found surveys using the Perryman method worked well for relatively small sub-colonies of around a thousand birds. Many large colonies of Pygoscelis penguins are divided into sub-colonies of this approximate size which would be amenable to survey, one-by-one, by this method until all are 
counted. However, some penguin colonies form continuous, dense carpets that cover several hectares and they would be best surveyed by a UAV with autopilot capability.

We surveyed a single-species colony, but the quality of the images would allow different species of penguin to be identified within mixed colonies. Colonies at which high densities of large flying birds occur could interfere with surveys, either owing to risk of collision with the UAV or them flying between the lens and the colony, obscuring penguins on the image. Our survey was conducted on a flat open area, and this habitat is typical of most Pygoscelis and Aptenodytes penguins. However, many crested penguin colonies (genus Eudyptes) nest on steep scree slopes and so transects over these by the UAV would need to allow for altitudinal variation, making the programming of waypoints or manual flight more challenging. The approach will be unsuitable for burrow or cavity nesting species (genus Spheniscus or Eudyptula) and for those Eudyptes species that breed under a canopy of trees or tussock grass.

The image analysis approach we adopted uses open source software and is very quick and simple to use. This was our intention as it makes UAV surveys of seabirds, from start to finish, accessible to anyone. The image stitching is so fast (a matter of a few minutes) that it could be done in the field to ensure that there are no gaps in coverage. Should any gaps be evident, a second mission could be flown to fill these. Where spatial attributes of colonies are of interest, analysis of geo-referenced images in ArcGIS is required rather than simple image stitching (Grenzdöffer 2013). The manual counting of images was effective in our example, but large colonies of penguins will be best counted automatically using image recognition methods (Trathan 2004; Grenzdöffer 2013).

Despite the limitations of UAVs, they are likely to have their place in the array of methods that can be used to count penguins in the future. We have shown that multi-rotor UAVs can be used for surveys over short durations and range, while larger fixed-wing UAVs have potential to survey large areas in a single mission (Hodgson et al. 2013). Of the count methods available, those from UAVs are likely to be the most accurate and so provide a gold-standard against which other count types can be 
calibrated. The various survey methods should therefore be regarded as complementary rather than competing approaches.

\section{Acknowledgements}

RSPB kindly granted permission to conduct test flights over their farm in the UK. We thank Buzzflyer for advice on, and supply of, the UAV equipment. Martin Collins generously accommodated NR during the fieldwork. Megan Tierney and Nathan McNally assisted with the fieldwork. Permission for fieldwork in the Falklands was provided by the Falkland Island Environment Committee and permission to access the site was granted by Jan Cheek of Johnson Harbour Farm.

\section{References}

Anderson, K. and Gaston, K.J. 2013. Lightweight unmanned aerial vehicles will revolutionize spatial ecology. Front. Ecol. Environ. 11:138-146.

Chabot, D. and Bird, D.M. 2013. Small unmanned aircraft: precise and convenient new tools for surveying wetlands. J. Unmanned Veh. Sys. 1: 15-24.

Fretwell, P.T., LaRue, M,A,, Morin, P., Kooyman, G.L., Wienecke, B., Ratcliffe, N., Fox, A.J., Fleming, A.H., Porter, C. and Trathan, P.N. 2012. An Emperor Penguin Population Estimate: The First Global, Synoptic Survey of a Species from Space. PLoS ONE, 7: e33751.

Fox News. 2012. Drone test for seabird survey delayed due to lack of permit. Available from http://www.foxnews.com/scitech/2012/07/26/drone-test-for-seabird-survey-delayed-due-tolack-permit/ [accessed 30 Jan 2015].

Gardner, S., LeRoi, D. and Perryman, W. 2010. A penguin population polar express: NOAA's quest to count penguin breeds speeds up with a VTOL UAS. Unmanned Systems, 29: 30-35. Available from http://www.academia.edu/6559126/A PENGUIN POPULATION POLAR EXPRESS [accessed 30 January 2015]. 
GIMP. 2015. GNU Image Manipulation Programme for Windows Version 2.8.14.

http://www.gimp.org/ [accessed 30 January 2015].

Goebel, M.E., Perryman, W.L., Hinke, J.T., Krause, D.J., Hann, N.A., Gardner, S. and LeRoi, D.J. 2015. A small unmanned aerial system for estimating abundance and size of Antarctic predators. Polar Biol. DOI 10.1007/S00300-014-1625-4.

Grenzdöffer, G.J. 2013. UAS-based automatic bird count of a common gull colony. International Archives of the Photogrammetry, Remote Sensing and Spatial Information Sciences, XL-1/W2: 169-174. Available from http://www.int-arch-photogramm-remote-sens-spatial-inf-sci.net/XL1-W2/169/2013/isprsarchives-XL-1-W2-169-2013.pdf [accessed 30 January 2015].

Hodgson, J. and Clark, R. 2014. Conservation drones for seabird monitoring. http://conservationdrones.org/2014/05/05/conservation-drones-for-seabird-monitoring/ [accessed 30 January 2015].

Hodgson, A., Kelly, N. and Peel, D. 2013. Unmanned aerial vehicles (UAVs) for surveying marine fauna: a dugong case study. PLoS ONE, 8: e79556.

Koh, L.P. and Wich, S.A. 2012. Dawn of drone ecology: low-cost autonomous aerial vehicles for conservation.Tropical Conservation Science, 5: 121-132.

Lynch, H.J., Naveen, R. and Fagan, W.F. 2008. Censuses of penguins, blue-eyed shags, and southern giant petrel populations in the Antarctic Peninsula, 2001-2007. Mar. Ornithol. 36:83-97.

Lynch, H.J., Naveen, R., Trathan, P.N. and Fagan, W.F. 2012a. Spatially integrated assessment reveals widespread changes in penguin populations on the Antarctic Peninsula. Ecology, 93:13671377.

Lynch, H.J., White, R., Black, A.D. and Naveen, R. 2012b. Detection, differentiation, and abundance estimation of penguin species by high-resolution satellite imagery. Polar Biol. 35:963-968.

Magic Lantern. 2014. http://www.magiclantern.fm/ [accessed 30 January 2015]. 
Marquis, S. 2013. NOAA testing whether unmanned aircraft can help manage Olympic Coast National Marine Sanctuary resources. Available from http://sanctuaries.noaa.gov/news/press/2013/pr062013.html [accessed 30 January 2015].

Merino, L., Caballero, F., Martínez-de-Dios, J. Maza, I. and Ollero, A. 2011. An unmanned aircraft system for automatic forest fire monitoring and measurement. J. Intell. Robot Syst. 65: $533-548$

Microsoft Research. 2011. Image Composite Editor Version 1.4.4. http://research.microsoft.com/enus/um/redmond/groups/ivm/ICE/ [accessed 30 January 2015].

Mulero-Pázmány, M., Stolper, R., van Essen, L.D., Negro, J.J. and Sassen, T. 2014. Remotely piloted aircraft systems as a rhinoceros anti-poaching tool in Africa. PLoS ONE, 9: e83873.

Points in Focus. 2010. Depth of field, angle and field of view and equivalent lens calculator. http://www.pointsinfocus.com/tools/depth-of-field-and-equivalent-lenscalculator/\#fmt=7andap=2andfl=22anddst=30andu=m [accessed 30 January 2015].

Sardà-Palomera, F., Bota, G., Viñolo, C., Pallarés, O., Sazatornil, V., Brotons, L., Gomáriz, S. and Sarda, F. 2012. Fine-scale bird monitoring from light unmanned aircraft systems. Ibis, 154: 177-183. Trathan, P.N., Ratcliffe, N. and Masden, E.A. 2012. Ecological drivers of change at South Georgia: the krill surplus, or climate variability. Ecography, 35:983-993.

Vermeulen, C., Lejeune, P., Lisein, J., Sawadogo, P. and Bouché, P. 2013. Unmanned aerial survey of elephants. PLoS ONE, 8: e54700.

Viquerat, S., and van Neer, A. 2014. iTag image tagging software manual V0.6.0.1. http://sourceforge.net/projects/itagbiology/ [accessed 30 January 2015].

Watts, A.C., Perry, J.H., Smith, S.E., Burgess, M.A., Wilkinson, B.E., Szantoi, Z., Ifju, P. and Percival, H.F. 2010. Small unmanned aircraft systems for low-altitude aerial surveys. J. Wildl. Manage. 7: 1614-1619. 
Figure 1: The Cinestar 6 hexacopter in flight with the Canon EOS-M camera mounted beneath. (Photo credit: Sarah Crofts).

Figure 2: A view of the Cinestar 6 hexacopter with operators and base station, with a gentoo penguin colony in the background. (Photo credit: Sarah Crofts).

Figure 3: A cropped image of gentoo penguins in the colony, showing the detail available in fullresolution images. All birds are incubating or brooding apart from the bird at the top of the image which is standing next to its partner. A chick can clearly be seen beneath the bird standing at the bottom of the image. (Photo credit: Norman Ratcliffe).

Figure 4: The stitched mosaic of images covering the entire colony from which counts were made. Note the positions of the two fieldworkers moving along the colony perimeter on successive transects (Photo credit: Norman Ratcliffe). 


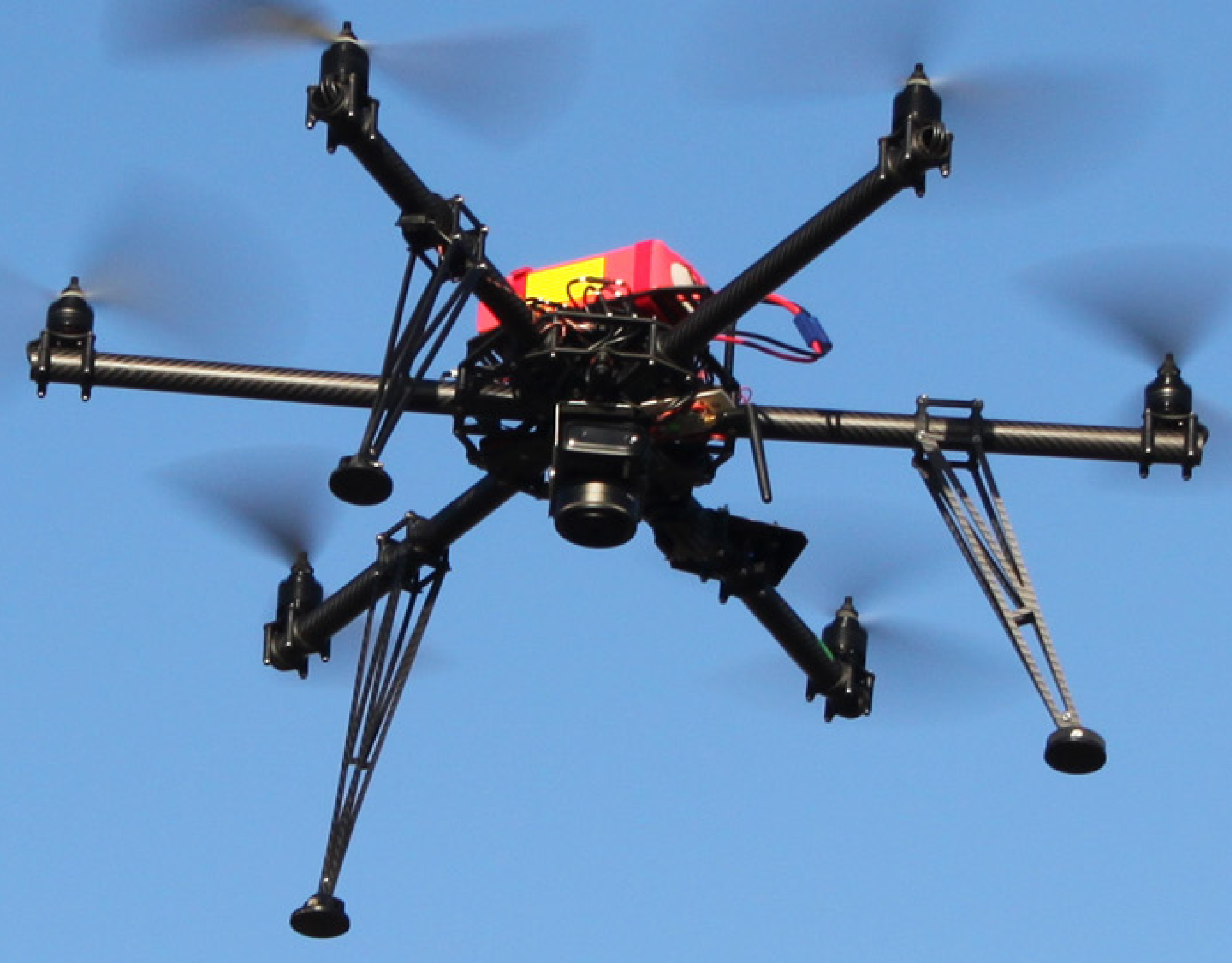




\section{एका}

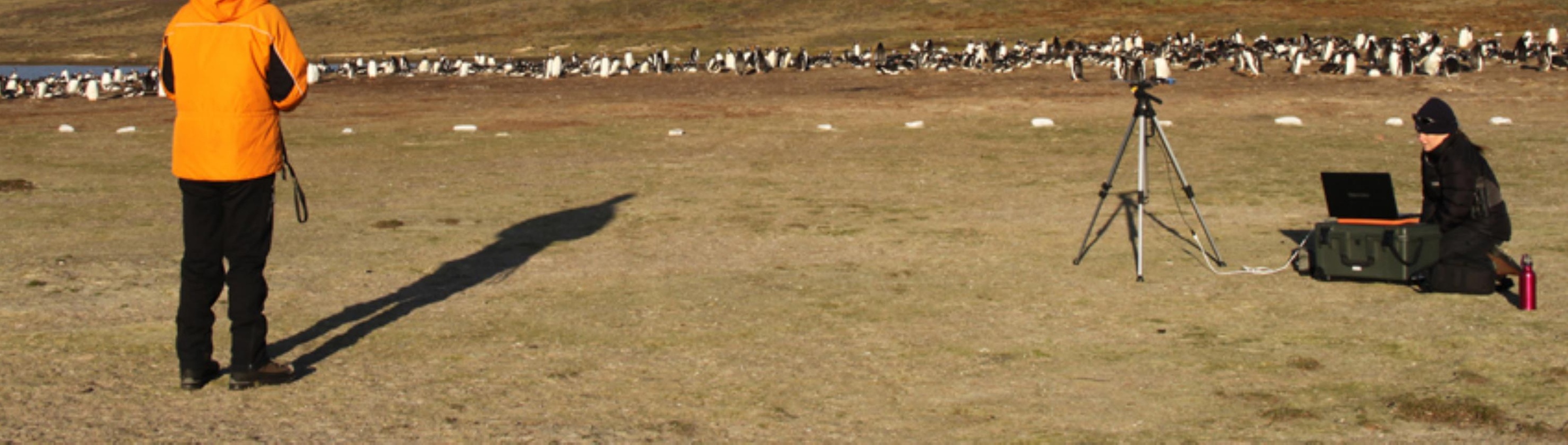


